\title{
A BI-OBJECTIVE SCORE-VARIANCE BASED LINEAR ASSIGNMENT METHOD FOR GROUP DECISION MAKING WITH HESITANT FUZZY LINGUISTIC TERM SETS
}

\author{
Seyed Hossein RAZAVI HAJIAGHA ${ }^{1 *}$, Meisam SHAHBAZI ${ }^{2}$, \\ Hannan AMOOZAD MAHDIRAJI ${ }^{3}$, Hossein PANAHIAN ${ }^{4}$ \\ ${ }^{1}$ Department of Management, Faculty of Management and Finance, Khatam University, Tehran, Iran \\ ${ }^{2}$ Faculty of management and accounting, Farabi Campus, University of Tehran, Iran \\ ${ }^{3}$ Faculty of Management Tehran, University of Tehran, IR \\ ${ }^{4}$ Department of Management, Kashan Branch, Islamic Azad University, Kashan, Iran
}

Received 10 September 2016; accepted 19 December 2016

\begin{abstract}
Decision makers usually prefer to express their preferences by linguistic variables. Classic fuzzy sets allowed expressing these preferences using a single linguistic value. Considering inevitable hesitancy of decision makers, hesitant fuzzy linguistic term sets allowed them to express individual evaluation using several linguistic values. Therefore, these sets improve the ability of humans to determine believes using their own language. Considering this feature, in this paper a method upon linear assignment method is proposed to solve group decision making problems using this kind of information, when criteria weights are known or unknown. The performance of the proposed method is illustrated in a numerical example and the results are compared with other methods to delineate the models efficiency. Following a logical and well-known mathematical logic along with simplicity of execution are the main advantages of the proposed method.
\end{abstract}

Keywords: linguistic variables, hesitant fuzzy linguistic term sets, multi-criteria group decision making, linear assignment method, National Cartographic Center.

JEL Classification: M02.

\section{Introduction}

Multi-criteria decision making (henceforward MCDM) is a well-known and widely used area of operational research. Classic operation research techniques usually determined the best decision considering only a single criterion. Considering multiple criteria is a tool for decreasing the risk of decisions. MADM has a wide variety of applications in different fields (Hwang, Yoon 1995; Tzeng, Huang 2011).

*Corresponding author. E-mail: s.hossein.r@gmail.com

This is an Open Access article distributed under the terms of the Creative Commons Attribution License (http://creativecommons. org/licenses/by/4.0/), which permits unrestricted use, distribution, and reproduction in any medium, provided the original author and source are credited. 
Formally speaking, Zimmermann (Zimmerman 1987) defined a multi-criteria decision making problem as follows. Let we have a nonempty and finite set of decision alternatives, i.e., $A_{1}, A_{2}, \ldots, A_{m}$, and there are a finite set of goals, attributes or criteria, i.e., $C_{1}, C_{2}, \ldots, C_{m}$, according to which the desirability of an alternative is to be judged. The aim of MADM is to determine the optimal alternative with the highest degree of desirability with respect to all relevant goals. An MADM problem usually is formulated in the form of a decision matrix, $D=\left[x_{i j}\right]$, where $x_{i j}$ illustrates the performance of the alternative $A_{i}$ regard to criterion $C_{j}$. $\mathrm{Yu}(\mathrm{Yu}$ 1990) stated that an MADM problem consists of four critical elements, including (1) alternatives, (2) decision criteria, (3) performance of alternatives with regard to criteria, and (4) the weight vector of criteria importance. Two main features of MADM problems are that decisions are usually adopted by a group of experts, and decision information is usually uncertain. The uncertainty is a result of partial and approximate information (Yu 1990). This uncertainty arose in the $3^{\text {rd }}$ and $4^{\text {th }}$ elements of an MADM problem.

Different frameworks are proposed for dealing with uncertainty. Liu and Lin (2011) classified these frameworks into three classes encompassing (1) probability and statistic, (2) fuzzy set theory, and (3) grey system theory. Fuzzy set theory (Zadeh 1965) is one of the mostly applied frameworks of dealing with uncertainty and the first work of decision making with fuzzy information dates back to Bellman and Zadeh (1970). Since its initial form of fuzzy sets, where a membership degree is assigned to elements of a set, different researchers tried to extend its applicability. Some scholars believe that assigning a single membership degree to an element, without considering decision makers' hesitancy, is a limitation of fuzzy sets (Zadeh 1975; Grattan-Guinness 1976). Therefore, scholars developed some new extensions of classic fuzzy sets, considering this hesitancy. Among these extensions can refer to type-2 fuzzy sets (Liang, Mendel 2000) where membership function illustrated itself as a fuzzy set, interval type-2 fuzzy sets (Atanassov 1986) that membership functions considered as closed intervals, intuitionistic fuzzy sets (Atanassov 1986) where a non-membership degree is also defined for each element along with its membership allowing a hesitant degree, intervalvalued intuitionistic fuzzy sets (Atanassov, Gargov 1989) where membership and non-membership degrees illustrated as closed intervals, and etc. These generalizations of fuzzy sets are applied extensively in decision making problems by Razavi Hajiagha et al. (2013, 2014a, 2014b, 2014c, 2015).

Hesitant fuzzy sets (henceforth HFS) (Torra 2010) are another form of fuzzy sets where several possible values are allowed for determining the membership of an element in the corresponding set. Usually, HFS is applied in quantitative situations, while sometimes uncertainty is due to vagueness of qualitative meanings rather than quantitative measures (Liao et al. 2014). In this case, Zadeh (1975) proposed the fuzzy linguistic approach. This concept was later extended to 2-tuple fuzzy linguistic model (Martinez, Herrera 2010; Herrera, Martinez 2000). A constraint of these approaches is expert's limitation in illustrating their preferences with a single linguistic term (Liao et al. 2014). Inspired from HFS, Rodríguez et al. (2012) proposed hesitant fuzzy linguistic term sets (hereafter HFLTSs), where experts can have expressed their judgments with several linguistic values. In this context, experts can express their preferences using comparative terms such as "between very low and medium". 
Compared with fuzzy linguistic approaches, HFLTSs are more convenient and flexible to reflect the Decision makers' preferences in decision making process ( $\mathrm{Zhu}, \mathrm{Xu} 2014$ ).

The first application of HFLTSs in decision making is presented by Rodríguez et al. (2012). Later, Rodríguez et al. (2013) proposed a new linguistic group decision model applying comparative linguistic expressions that is close to human cognitive models based on HFLTSs and context-free grammars. Liu and Rodríguez (2014) employed a fuzzy envelope for computing with words and applied it in MADM. Liao et al. (2014) proposed different similarity and distance measures among HFLTSs and introduced the notions of hesitant fuzzy linguistic positive and negative solutions for MADM. Zhu and Xu (2014) developed several consistency measures for HFLPRs to make sure that the Decision makers are being neither random nor illogical. Wang et al. (2015) introduced an outranking approach, similar to ELECTRE, for MADM with HFLTSs. Farhadnia (2015) extended the notion of Entropy for finding criteria weights under HFLTSs. Lee and Chen (2015) recommended a group decision making method on the basis of likelihood-based comparison relations of HFLTSs and introduced some operators on these sets. Liao et al. (2015a) used HFLTSs with VIKOR method and its application in the process of qualitative decision making qualitative multiple criteria decision making. Liao et al. (2015b) examined the correlation measures and coefficients between HFLTSs and proposed its application in the process of qualitative decision making. Liao and Xu (2015) used the cosine-distance-based HFL-TOPSIS method and the cosine-distance-based HFL-VIKOR method for selecting an ERP system. Montes et al. (2015) designed a web tool for decision making in housing market using HFLTSs. Wang and $\mathrm{Xu}$ (2015) believe that extended hesitant fuzzy linguistic term sets (EHFLTSs) are a useful method for modeling the uncertain linguistic data in group decision making.

Wei et al. (2015) extended the TODIM method of MADM under HFLTSs. Furthermore, some researchers focused on hesitant fuzzy linguistic preference relations as a new preference structure and extended their applications in MCDM (Zhang, Wu 2014; Wu, Xu 2015). Zhu and $\mathrm{Xu}$ (2016) extended EHFSs by combining the proposed distance measures with the Dempster-Shafer belief structure.

The aim of this paper is to develop a new form of linear assignment method (LAM) for MADM when expert's judgments are expressed according to HFLTSs. This method is extended under two different conditions when (1) criteria weights are predetermined and (2) criteria weights are unknown. The main motivation beyond extending LAM under HFLTS is firstly due to simplicity and clear logic of LAM method; secondly due to abilities of HFLTS in capturing decision makers uncertainty; and thirdly providing an efficient method of dealing with HFLTS in real world applications.

Bernardo and Blin (1977) initially scheduled LAM method inspiring from assignment problem in linear programming for MADM. Classic LAM method relies on crisp values of decision matrix. Considering inevitable uncertainty of decision making problems, LAM is extended under different uncertainty frameworks; moreover, fuzzy LAM method is extended by scholars using different modelling and solving approaches (Lin, Wen 2004; Bashiri et al. 2011; Baykasoğlu et al. 2016). Lin and Wen (2004) applied the min-max criterion of Bellman and Zadeh (1970) and formulated the fuzzy LAM method in the form of a mixed integer nonlinear programming problem. Bashiri et al. (2011) aggregated the judgments of decision 
makers by averaging their opinions and then, they solved a linear programming problem to find the optimal permutation of the alternatives. Then, they used the obtained permutation in subsequent linear programming models to find the final ranking of alternatives. Baykasoğlu et al. (2016) applied various concepts of fuzzy sets theory such as fuzzy arithmetic and aggregation, fuzzy ranking and fuzzy mathematical programming to solve a MADM problem using fuzzy LAM. Chen (2013) extended the LAM under interval type-2 fuzzy sets. The interval valued intuitionistic fuzzy LAM is also proposed by Chen (2014). Zamri and Abdullah (2015) used the linear assignment method and simple additive weighting through the type-2 Fuzzy sets proposed a robust and user-friendly method in decision making framework. Abdolazimi et al. (2015) used linear assignment method in zoning Shahroud-Bastam watershed for artificial recharge of groundwater for GIS technique and compared it with ELECTRE results. They found that the results of linear assignment method are more accurate and are more consistent with reality.

The need for presenting a new approach of decision making with HFLTS can be justified due to reasonable requirements. First, the flexibility and ability of HFLTS in describing decision makers' ambiguity made it as an interesting tool for decision making problems under uncertainty. Second, considering non-existence of an exact solution for uncertain problems (referred to as an axiom in (Zadeh 1965), it seems appropriate empowering analysts with more tools for dealing with uncertain decision making problems. Third, the clear and simple logic behind linear assignment method made it as a fascinating candidate for extending it under different uncertainty frameworks. Accordingly, upon above reasons, in this paper the LAM method is extended for group decision making problems with HFLTS information.

This contribution is structured as follows; first of all, a brief overview on required notions of HFLTSs is given in section 1. The modelling and solving the linear assignment method with HFLTSs is detailed totally in section 2 with known and unknown weights. A numerical example is then solved via the advanced method and the results are compared with other methods. Finally, conclusion remarks are given in section Conclusions.

\section{Hesitant fuzzy linguistic term sets}

Linguistic variables are variables whose values are words or sentences rather than values (Zadeh 1975). Using linguistic variables is a more realistic approach since it is closer to human cognition (Farhadnia 2015; Herrera et al. 1996). As a case in point, criteria such as age of a person, speed of a car, story of a movie and etc. can be described easily applying linguistic variables. There are different approaches to select linguistic descriptors and define their semantics. With this fact in mind, in this section an overview is represented on the notion of HFLTS.

Definition 1.1. (Torra 2010). Let $X$ be a fixed set, a hesitant fuzzy set on $X$ is in terms of a function $h$ that when applied to $X$ returns a subset of $[0,1]$.

$\mathrm{Xia}$ and $\mathrm{Xu}$ (2011) defined a hesitant fuzzy set mathematically as

$$
E=\left\{x, h_{E}(x) \mid x \in X\right\},
$$

where $h_{E}(x)$ is a set of values in $[0,1]$. 
HFS can be applied on quantitative variables. Rodríguez et al. (2012) introduced the notion of HFLTSs for qualitative variables.

Definition 1.2. Let $S$ be a linguistic term set, $S=\left\{s_{0}, \ldots, s_{g}\right\}$, a HFLTS, $H_{S}$, is an ordered finite subset of the consecutive linguistic terms of $S$.

The commonly used approaches for selecting linguistic descriptors include the ordered structure approach and context-free grammar approach (Rodríguez et al. 2012). For example, $S$ can be defined as $S=\left\{s_{0}\right.$ : nothing, $s_{1}$ : very low, $s_{2}$ : low, $s_{3}$ : medium, $s_{4}$ : high, $s_{5}$ : very high, $s_{6}$ : perfect $\}$. A HFLTS can be defined as $H_{s}(\vartheta)=\left\{s_{1}, s_{2}, s_{3}\right\}$.

A totally ordered discrete linguistic term set is proposed by $\mathrm{Xu}$ (Xu 2004, 2005, 2012] as $\sigma=\left\{s_{\alpha} \mid \alpha=-\tau, \ldots,-1,0,1, \ldots, \tau\right\}$ where $\tau$ is a positive integer $s_{\alpha}$ represent a possible value for a linguistic variable; thus, $S$ satisfies the following conditions:

(1) If $\alpha>\beta$, then $s_{\alpha}>s_{\beta}$.

(2) The negation operator is defined as: $\operatorname{neg}\left(s_{\alpha}\right)=s_{-\alpha}$, especially, $\operatorname{neg}\left(s_{0}\right)=s_{0}$.

For $\tau=3, S$ can be taken as: $S=\left\{s_{-3}=\right.$ none, $s_{-2}=$ very low, $s_{-1}=$ low, $s_{0}=$ medium, $s_{1}=$ high, $s_{2}=$ very high, $s_{3}=$ perfect $\}$. A similar scale of this 7 points is represented by Farhadnia (2015) for appraising the speed of a car. Inspiring from mathematical definition of HFS by Xia and Xu (2011), Liao et al. (2014) redefined the concept of HFLTS mathematically as below (restated by Farhadnia (2015).

Definition 1.3. Let $X=\left\{x_{1}, x_{2}, \ldots, x_{N}\right\}$ beareferenceset, and $\sigma=\left\{s_{\alpha} \mid \alpha=-\tau, \ldots,-1,0,1, \ldots, \tau\right\}$ be a linguistic term set. A HFLTS on $X$ is mathematically shown in terms of

$$
H_{\sigma}=\left\{x_{i}, h_{\sigma}\left(x_{i}\right) \mid x_{i} \in X\right\},
$$

where, $h_{\sigma}\left(x_{i}\right)$ is a set of some possible values in the linguistic term set $\sigma$. To adjust the notion of HFLTS, consider the following example.

Example 1.4. (Farhadnia 2015) Suppose that an expert approximate speed of three cars $x_{1}, x_{2}$, and $x_{3}$. This expert believes that $x_{1}$ speed is "at least fast", $x_{2}$ speed is "between very slow and average", and $x_{3}$ speed as "great than fast". Considering the following term set $S=\left\{s_{-3}=\right.$ very slow, $s_{-2}=$ slow, $s_{-1}=$ slightly slow, $s_{0}=$ average, $s_{1}=$ slightly fast, $s_{2}=$ fast, $s_{3}=$ very fast $\}$, the HFLTS of appraising these cars can be shown as $\left\{x_{1}, h_{\sigma}\left(x_{1}\right)=\left\{s_{2}, s_{3}\right\}, x_{2}, h_{\sigma}\left(x_{2}\right)=\left\{s_{-3}, s_{-2}, s_{-1}, s_{0}\right\}, x_{3}, h_{\sigma}\left(x_{3}\right)=\left\{s_{3}\right\}\right\}$.

For ranking HFLTSs, the notion of HFLTS score function is introduced (Herrera et al. 1996).

Definition 1.5. For a HFLTS defined over linguistic term set $\sigma=\left\{s_{\alpha} \mid \alpha=-\tau, \ldots,-1,0,1, \ldots, \tau\right\}$, where $h_{\sigma}=\left\{s_{\delta_{l}} \mid s_{\delta_{l}} \in \sigma, l=1,2, \ldots, L\right\}$, the score function $s\left(h_{\sigma}\right)$ is defined as:

$$
s\left(h_{\sigma}\right)=\frac{1}{L} \sum_{l=1}^{L} \delta_{l} .
$$

Liao et al. (2014) also defined the variance function of a HFS. Later, Farhadnia (2015) extended the following definition for variance function of HFLTS.

Definition 1.1. For HFLTS $h_{\sigma}$ defined according to definition 2.4, its variance function is defined as:

$$
v\left(h_{\sigma}\right)=\sqrt{\frac{1}{(L)_{2}} \sum_{l \neq k=1}^{L}\left(\delta_{l}-\delta_{k}\right)} .
$$


If $h_{1}(\sigma)$ and $h_{2}(\sigma)$ are two HFLTSs, then:

- If $s\left(h_{1}\right)>s\left(h_{2}\right)$, then $h_{1}(\sigma)>h_{2}(\sigma)$;

- If $s\left(h_{1}\right)=s\left(h_{2}\right)$, then

1. If $v\left(h_{1}\right)=v\left(h_{2}\right)$, then $h_{1}(\sigma) \approx h_{2}(\sigma)$;

2. If $v\left(h_{1}\right)>v\left(h_{2}\right)$, then $h_{1}(\sigma)<h_{2}(\sigma)$;

3. If $v\left(h_{1}\right)<v\left(h_{2}\right)$, then $h_{1}(\sigma)>h_{2}(\sigma)$.

\section{Linear assignment method with HFLTSs}

Classic linear assignment method (LAM) is proposed by Bernardo and Blin (1977). In this section, LAM is extended in situations where a group of experts expressed their opinions on HFLTSs. Considering a group of $K$ experts, $e_{k}, k=1,2, \ldots, K$ participated in a group decision making problem, where a finite set of alternatives $A=\left\{A_{1}, A_{2}, \ldots, A_{m}\right\}$ are evaluated upon a finite set of criteria $C=\left\{c_{1}, c_{2}, \ldots, c_{n}\right\}$. Preferences of experts are stated in a linguistic term set $\sigma=\left\{s_{\alpha} \mid \alpha=-\tau, \ldots,-1,0,1, \ldots, \tau\right\}$. Each expert $k$ expressed his opinion about performance of alternative $A_{i}$ regard to criterion $c_{j}$ using a HFLTS $h_{k}^{i j}(\sigma)$; therefore, at the first step, individual decision matrices are obtained as:

$$
D_{k}=\left[\begin{array}{cccc}
h_{k}^{11}(\sigma) & h_{k}^{12}(\sigma) & \cdots & h_{k}^{1 n}(\sigma) \\
h_{k}^{21}(\sigma) & h_{k}^{22}(\sigma) & \cdots & h_{k}^{2 n}(\sigma) \\
\vdots & \vdots & \ddots & \vdots \\
h_{k}^{m 1}(\sigma) & h_{k}^{m 2}(\sigma) & \cdots & h_{k}^{m n}(\sigma)
\end{array}\right] .
$$

It is natural that decision makers have different opinions about elements of decision matrix. For instance, considering linguistic term set $S=\left\{s_{-3}=\right.$ none, $s_{-2}=$ very low, $s_{-1}=$ low, $s_{0}=$ medium, $s_{1}=$ high, $s_{2}=$ very high, $s_{3}=$ perfect $\}$, one decision maker may believe that alternative $A_{i}$ performed "perfect" at criterion $c_{j}$ (denoted as $h_{1}^{i j}=\left\{s_{3}\right\}$ ), while the other decision maker appraise its performance as "between medium to very high" (denoted as $\left.h_{2}^{i j}=\left\{s_{0}, s_{1}, s_{2}\right\}\right)$. If they can persuade each other, an aggregated HFLTS is obtained. Otherwise, their aggregated assessment can be represented as $h^{i j}=\left\{s_{0}, s_{1}, s_{2}, s_{3}\right\}$. This method of aggregation is proposed and applied by Liao et al. (2014), Farhadnia (2015), the logic behind this aggregation operator is designed based on negotiation, consensus and persuasion without any value given to a specific decision maker. Hence, in this step, an aggregated decision matrix is composed.

$$
D_{k}=\left[\begin{array}{cccc}
h^{11}(\sigma) & h^{12}(\sigma) & \cdots & h^{1 n}(\sigma) \\
h^{21}(\sigma) & h^{22}(\sigma) & \cdots & h^{2 n}(\sigma) \\
\vdots & \vdots & \ddots & \vdots \\
h^{m 1}(\sigma) & h^{m 2}(\sigma) & \cdots & h^{m n}(\sigma)
\end{array}\right] .
$$

Now, the score function of decision matrix elements is computed to form the score function decision matrix as: 


$$
S\left(D_{k}\right)=\left[\begin{array}{cccc}
s\left(h^{11}\right) & s\left(h^{12}\right) & \cdots & s\left(h^{1 n}\right) \\
s\left(h^{21}\right) & s\left(h^{22}\right) & \cdots & s\left(h^{2 n}\right) \\
\vdots & \vdots & \ddots & \vdots \\
s\left(h^{m 1}\right) & s\left(h^{m 2}\right) & \cdots & s\left(h^{m n}\right)
\end{array}\right]
$$

Similarly, adopting variance function definition of HFLTS, the variance function decision matrix is constructed as:

$$
V\left(D_{k}\right)=\left[\begin{array}{cccc}
v\left(h^{11}\right) & v\left(h^{12}\right) & \cdots & v\left(h^{1 n}\right) \\
v\left(h^{21}\right) & v\left(h^{22}\right) & \cdots & v\left(h^{2 n}\right) \\
\vdots & \vdots & \ddots & \vdots \\
v\left(h^{m 1}\right) & v\left(h^{m 2}\right) & \cdots & v\left(h^{m n}\right)
\end{array}\right] .
$$

Afterwards, considering the comparison role given at the last paragraph of section 2, data for criteria in both $S\left(D_{k}\right)$ and $V\left(D_{k}\right)$ can be used to formulate a bi-objective linear assignment model. The values of score functions in a given criterion $j$, i.e., $\left\{s\left(h^{1 j}\right), s\left(h^{2 j}\right), \ldots, s\left(h^{m j}\right)\right\}$, can be ranked in a descending manner while variance functions, i.e., $\left\{v\left(h^{1 j}\right), v\left(h^{2 j}\right), \ldots, v\left(h^{m j}\right)\right\}$, are ranked in an ascending manner. This modeling proceeds considering the situation whether criteria weight vector $w=\left(w_{1}, w_{2}, \ldots, w_{n}\right)$ is predetermined or unknown.

\subsection{LAM with predetermined weights}

First, let the criteria weight vector $w=\left(w_{1}, w_{2}, \ldots, w_{n}\right)$ be known. These weights can be obtained via subjective judgments, pairwise comparisons (Saaty 1977), Entropy (Hwang, Yoon 1995), LINMAP (Srinivasan, Shocker 1973), factor relation (Ginevičius 2011), or any other method. In this way, suppose that an alternative $A_{i}$ takes rank $l, l \in\{1,2, \ldots, m\}$ in $j$ th criterion on score function and takes rank $l^{\prime}, l^{\prime} \in\{1,2, \ldots, m\}$ upon variance function. The set of all criteria in which $A_{i}$ takes rank $l$ is denoted as $C$.

Correspondingly, if alternative $A_{i}$ finally occupies the rank $l$, this decision's utility will be $w_{l}^{i}=\sum_{j \in C^{l}} w_{j}$. Then as well, for variance function if $A_{i}$ finally occupies the rank $l^{\prime}$, this decision's utility on variance function will be $w_{l}^{\prime i}=\sum_{j \in C^{l}} w_{j}$. Considering ranking of all alternatives over all criteria's, $S\left(D_{k}\right)$ and $V\left(D_{k}\right)$ are transformed into the following two matrices, respectively,

$$
\Pi(S)=\left[\begin{array}{cccc}
w_{1}^{1} & w_{2}^{1} & \cdots & w_{m}^{1} \\
w_{1}^{2} & w_{2}^{2} & \cdots & w_{m}^{2} \\
\vdots & \vdots & \ddots & \vdots \\
w_{1}^{m} & w_{2}^{m} & \cdots & w_{m}^{m}
\end{array}\right]
$$


and

$$
\Pi^{\prime}(S)=\left[\begin{array}{cccc}
w_{1}^{\prime 1} & w_{2}^{\prime 1} & \cdots & w_{m}^{1} \\
w_{1}^{\prime 2} & w_{2}^{\prime 2} & \cdots & w_{m}^{\prime 2} \\
\vdots & \vdots & \ddots & \vdots \\
w_{1}^{\prime m} & w_{2}^{\prime m} & \cdots & w_{m}^{\prime m}
\end{array}\right]
$$

The optimal decision in this case can be determined by solving the below mathematical problem:

$$
\begin{aligned}
& \max \sum_{i=1}^{m} \sum_{l=1}^{m} w_{l}^{i} p_{i l}, \\
& \max \sum_{i=1}^{m} \sum_{l=1}^{m} w_{l}^{\prime i} p_{i l} \\
& \text { S.T. } \\
& \sum_{i=1}^{m} p_{i l}=1, i=1,2, \ldots, m ; \\
& \sum_{l=1}^{m} p_{i l}=1, l=1,2, \ldots, m ; \\
& p_{i l} \in\{0,1\}, i, l=1,2, \ldots, m .
\end{aligned}
$$

To solve this bi-objective assignment problem, a goal programming approach is suggested. First, two simple linear assignment models are solved by considering different objectives of Eq. (11). These models are demonstrated in the following relations:

$$
\max \sum_{i=1}^{m} \sum_{l=1}^{m} w_{l}^{i} p_{i l}
$$

S.T.

$$
\begin{aligned}
& \sum_{i=1}^{m} p_{i l}=1, l=1,2, \ldots, m ; \\
& \sum_{l=1}^{m} p_{i l}=1, i=1,2, \ldots, m ; \\
& p_{i l} \in\{0,1\}, i, l=1,2, \ldots, m .
\end{aligned}
$$

$$
\max \sum_{i=1}^{m} \sum_{l=1}^{m} w_{l}^{\prime i} p_{i l}
$$

S.T.

$$
\begin{aligned}
& \sum_{i=1}^{m} p_{i l}=1, l=1,2, \ldots, m ; \\
& \sum_{l=1}^{m} p_{i l}=1, i=1,2, \ldots, m ; \\
& p_{i l} \in\{0,1\}, i, l=1,2, \ldots, m .
\end{aligned}
$$

Suppose that solving Eq. (12), the optimal solution obtained as $S^{*}$ and the optimal solution of Eq. (13) be $V^{*}$. Therefore, to find the final solution of initial decision making problem, the following goal programming model is formulated: 


$$
\min d_{1}^{-}+d_{2}^{-}
$$

S.T.

$$
\begin{aligned}
& \sum_{i=1}^{m} \sum_{l=1}^{m} w_{l}^{i} p_{i l}+d_{1}^{-}-d_{1}^{+}=S^{*} ; \\
& \sum_{i=1}^{m} \sum_{l=1}^{m} w_{l}^{\prime i} p_{i l}+d_{2}^{-}-d_{2}^{+}=V^{*} ; \\
& \sum_{i=1}^{m} p_{i l}=1, i=1,2, \ldots, m ; \\
& \sum_{l=1}^{m} p_{i l}=1, l=1,2, \ldots, m ; \\
& p_{i l} \in\{0,1\}, i, l=1,2, \ldots, m ;
\end{aligned}
$$

Solving the problem in Eq. (14), the values of $p_{i l}=1$, for all $i, i=1,2, \ldots, m$ and $l, l=1,2, \ldots, m$ determine the ranking of alternative $A_{i}, i=1,2, \ldots, m$.

\subsection{LAM with unknown weights}

Occasionally, decision makers don't have sufficient information to determine the weight vector $w$. In this case, before constructing matrices of $\Pi(S)$ and $\Pi^{\prime}(S)$, determining $w$ is required. Confronting with this problem, the Entropy-based methods can be used to extract the weighting vector $\mathrm{w}$ from decision matrix directly. Farhadnia (2015) introduced a family of Entropy measures that is performable to determine criteria weights. E.g. his Entropy measure based on generalized distance is defined as bellow:

$$
E_{d_{g}}\left(H_{\sigma}\right)=1-\frac{2}{m} \sum_{i=1}^{m}\left[\left(\frac{1}{L} \sum_{l=1}^{L}\left(\frac{\left|\delta_{l}\right|}{2 \tau}\right)^{\lambda}\right)^{\frac{1}{\lambda}}\right] .
$$

Considering $\lambda=1$, the Entropy-based weights of criteria can be obtained as:

$$
w_{j}=\frac{1-E_{j}}{m-\sum_{j=1}^{m} E_{j}} .
$$

Farhadnia (2015) also determined different Entropy measures hinged on different distance measures, like generalized Hausdorff distance, hybrid Hamming distance, and etc. being applicable to determine the weight vector as well. These weights are applied for decision making based on LAM method, as described in previous section. 


\subsection{LAM with HFLTSs algorithm}

In this section, the linear assignment method for multi-criteria group decision making under HFLTSs is presented.

Step 1. Identify the alternatives set $A=\left\{A_{1}, A_{2}, \ldots, A_{m}\right\}$ and criteria set $C=\left\{c_{1}, c_{2}, \ldots, c_{n}\right\}$.

Step 2. If the weight vector $w=\left(w_{1}, w_{2}, \ldots, w_{n}\right)$ is determined, go to step 3 , otherwise go to step 9.

Step 3. Using Eqs (3) and (4), respectively, construct the score decision matrix, Eq. (7), and variance decision matrix, Eq. (8).

Step 4. Rank columns of score decision matrix in a decreasing manner and variance decision matrix columns in an increasing manner.

Step 5. Compute $w_{l}^{i}=\sum_{j \in C^{l}} w_{j}$ and $w_{l}^{\prime i}=\sum_{j \in C^{l}} w_{j}$ for alternative $A_{i}$ if it occupies the rank $l$ and $l^{\prime}$ in criteria of $C^{l}$ in score matrix and $C^{l^{\prime}}$ in variance matrix.

Step 6. Construct $\Pi(S)$, Eq. (9), and $\Pi^{\prime}(S)$, Eq. (10).

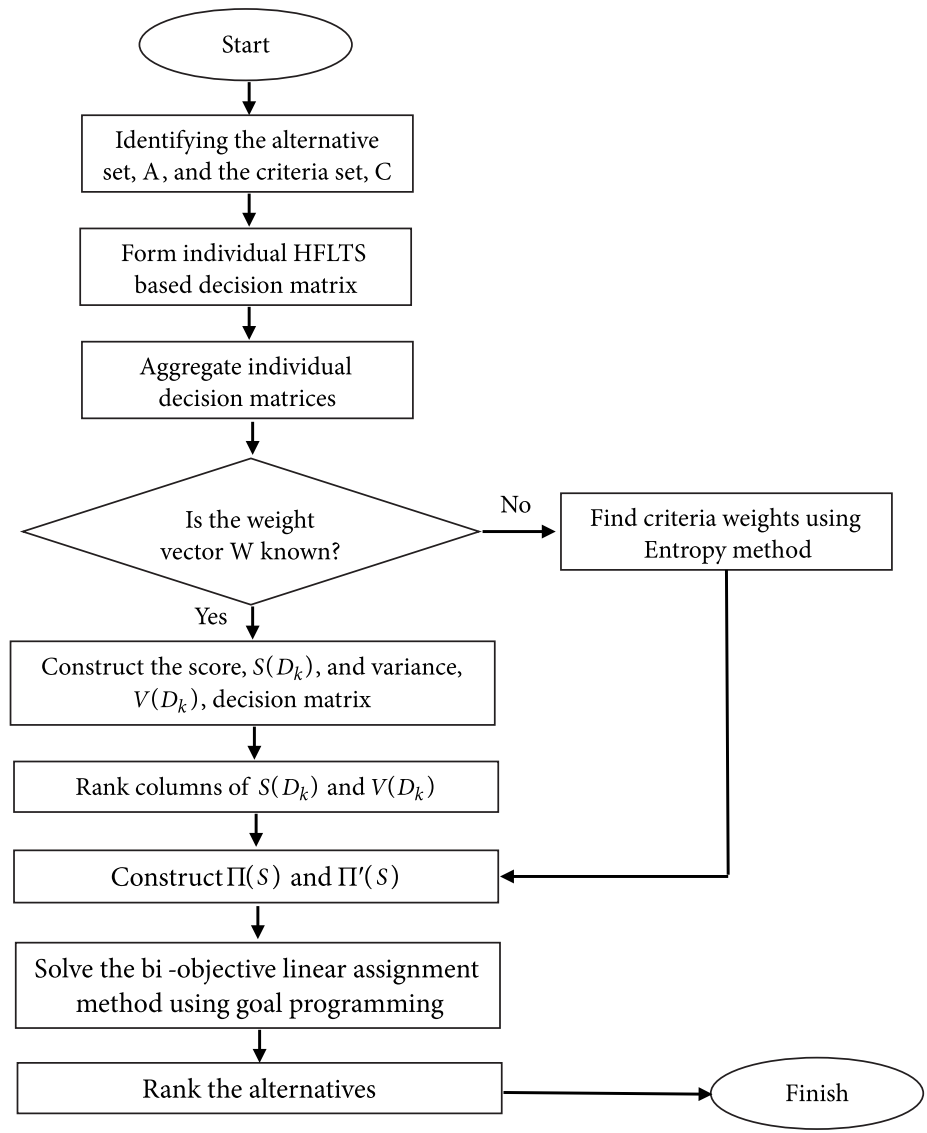

Fig. 1. Linear assignment method by HFLTSs 
Step 7. Formulate and solve two linear assignment problems based on Eqs (12) and (13). Step 8. Formulate and solve the goal programming problem of Eq. (14) to determine multi-criteria decision making problem's solution.

Step 9. Use Farhadnia (Farhadnia 2015) Entropy-based method to determine the weights of criteria and back to step 2 .

Figure 1 illustrates the flowchart of this algorithm.

\section{Numerical example}

In this section, an example is solved when criteria weight vector is predetermined. Liao et al. (2014) investigated the problem of a company evaluating five movies $x_{1}, x_{2}, \ldots, x_{5}$ based on four criteria encompassing story $\left(c_{1}\right)$, acting $\left(c_{2}\right)$, visuals $\left(c_{3}\right)$ and direction $\left(c_{4}\right)$. They proposed the weighting vector of these four criteria as $w=(0.4,0.2,0.2,0.2)$. The company expanded the seven point linguistic term set

$\sigma=\left\{s_{-3}=\right.$ terrible, $s_{-2}=$ very bad, $s_{-1}=$ bad, $s_{0}=$ medium, $s_{1}=$ well, $s_{2}=$ very well, $s_{3}=$ perfect\}. If decision makers in the company have different opinions about performance of a movie over a given criterion, they try to persuade each other, and if an aggregation isn't achieving, their judgments will be aggregated, as described in section 2 . Table 1 presents the HFLTSs decision matrix provided by the company.

Table 1. The hesitant fuzzy linguistic judgment matrix provided by decision organization

\begin{tabular}{|c|c|c|c|c|}
\hline Movie & $c_{1}$ & $c_{2}$ & $c_{3}$ & $c_{4}$ \\
\hline$m_{1}$ & $\left\{s_{-2}, s_{-1}, s_{0}\right\}$ & $\left\{s_{0}, s_{1}\right\}$ & $\left\{s_{0}, s_{1}, s_{2}\right\}$ & $\left\{s_{1}, s_{2}\right\}$ \\
\hline$m_{2}$ & $\left\{s_{0}, s_{1}, s_{2}\right\}$ & $\left\{s_{1}, s_{2}\right\}$ & $\left\{s_{0}, s_{1}\right\}$ & $\left\{s_{0}, s_{1}, s_{2}\right\}$ \\
\hline$m_{3}$ & $\left\{s_{2}, s_{3}\right\}$ & $\left\{s_{1}, s_{2}, s_{3}\right\}$ & $\left\{s_{1}, s_{2}\right\}$ & $\left\{s_{2}\right\}$ \\
\hline$m_{4}$ & $\left\{s_{0}, s_{1}, s_{2}\right\}$ & $\left\{s_{-1}, s_{0}, s_{1}\right\}$ & $\left\{s_{1}, s_{2}, s_{3}\right\}$ & $\left\{s_{1}, s_{2}\right\}$ \\
\hline$m_{5}$ & $\left\{s_{-1}, s_{0}\right\}$ & $\left\{s_{0}, s_{1}, s_{2}\right\}$ & $\left\{s_{0}, s_{1}, s_{2}\right\}$ & $\left\{s_{0}, s_{1}\right\}$ \\
\hline
\end{tabular}

Considering HFLTS matrix in Table 2, the score and variance decision matrix are obtained as illustrated in Tables 2 and 3, respectively.

Table 2. Score function decision matrix

\begin{tabular}{|c|c|c|c|c|}
\hline Movie & $c_{1}$ & $c_{2}$ & $c_{3}$ & $c_{4}$ \\
\hline$m_{1}$ & -1 & $1 / 3$ & 1 & $3 / 2$ \\
\hline$m_{2}$ & 1 & $3 / 2$ & $1 / 2$ & 1 \\
\hline$m_{3}$ & $5 / 3$ & 2 & $3 / 2$ & 2 \\
\hline$m_{4}$ & 1 & 0 & 2 & $3 / 2$ \\
\hline$m_{5}$ & $-1 / 3$ & 1 & 1 & $1 / 3$ \\
\hline
\end{tabular}


Table 3. Variance function decision matrix

\begin{tabular}{|c|c|c|c|c|}
\hline Movie & $c_{1}$ & $c_{2}$ & $c_{3}$ & $c_{4}$ \\
\hline$m_{1}$ & 1.41 & 1 & 1.41 & 1 \\
\hline$m_{2}$ & 1.41 & 1 & 1 & 1.41 \\
\hline$m_{3}$ & 1 & 1.41 & 1 & 0 \\
\hline$m_{4}$ & 1.41 & 1.41 & 1.41 & 1 \\
\hline$m_{5}$ & 1 & 1.41 & 1.41 & 1 \\
\hline
\end{tabular}

Consequent to Table 2, alternatives ranking in different criteria are obtained according to Table 4.

Table 4. Ranking alternatives based on score function

\begin{tabular}{|c|c|c|c|c|}
\hline Movie & $c_{1}$ & $c_{2}$ & $c_{3}$ & $c_{4}$ \\
\hline$m_{1}$ & 5 & 4 & 3 & 2 \\
\hline$m_{2}$ & 2 & 2 & 5 & 4 \\
\hline$m_{3}$ & 1 & 1 & 2 & 1 \\
\hline$m_{4}$ & 2 & 5 & 1 & 5 \\
\hline$m_{5}$ & 4 & 3 & 3 & \\
\hline
\end{tabular}

Similarly, ranking upon variance matrix, Table 3, obtained as:

Table 5. Ranking alternatives based on variance function

\begin{tabular}{|c|c|c|c|c|}
\hline Movie & $c_{1}$ & $c_{2}$ & $c_{3}$ & $c_{4}$ \\
\hline$m_{1}$ & 2 & 1 & 2 & 2 \\
\hline$m_{2}$ & 2 & 1 & 1 & 3 \\
\hline$m_{3}$ & 1 & 2 & 1 & 1 \\
\hline$m_{4}$ & 2 & 2 & 2 & 2 \\
\hline$m_{5}$ & 1 & 2 & 2 & 2 \\
\hline
\end{tabular}

Regarding Tables 4 and 5, the following two matrices are constructed.

$$
\Pi(s)=m_{3}\left[\begin{array}{c}
m_{1} \\
m_{5}
\end{array}\left[\begin{array}{ccccc}
0 & 0.1 & 0.1 & 0.2 & 0.4 \\
0 & 0.4 & 0 & 0.2 & 0.2 \\
0.8 & 0.2 & 0 & 0 & 0 \\
0.2 & 0.3 & 0 & 0 & 0.2 \\
0 & 0 & 0.3 & 0.4 & 0.2
\end{array}\right] .\right.
$$

Consider the value of 0.3 at $5^{\text {th }}$ row and third column of the above matrix. Conforming to Table $4, m_{5}$ occupied 3 rd rank at $c_{2}$ alone, and at $c_{3}$ along with $m_{1}$. Those, if $m_{5}$ ranked 
3 finally, it gains 0.2 from $c_{2}$ and dividing the weight of $c_{3}$ with $m_{1}$, it achieves a gain of 0.1 from $c_{3}$. Therefore, $\mathrm{w}_{3}^{5}=0.2+0.1=0.3$. Equivalently, for variance matrix the $\bigoplus^{\prime}(\mathrm{S})$ is constructed.

$$
\Pi^{\prime}(s)=m_{3}\left[\begin{array}{ccccc}
m_{4} \\
m_{5}
\end{array}\left[\begin{array}{ccccc}
0.1 & 0.216 & 0 & 0 & 0 \\
0.2 & 0.133 & 0.2 & 0 & 0 \\
0.5 & 0.066 & 0 & 0 & 0 \\
0 & 0.333 & 0 & 0 & 0 \\
0.2 & 0.2 & 0 & 0 & 0
\end{array}\right] .\right.
$$

Now, the problem in Eq. (12) is formulated as:

$$
\begin{aligned}
& \max 0 p_{11}+0.1 p_{12}+\ldots+0.4 p_{54}+0.2 p_{55} \\
& \text { S.T. } \\
& p_{11}+p_{12}+\ldots+p_{15}=1 ; \\
& p_{21}+p_{22}+\ldots+p_{25}=1 ; \\
& \vdots \\
& p_{51}+p_{52}+\ldots+p_{55}=1 ; \\
& p_{11}+p_{21}+\ldots+p_{51}=1 ; \\
& p_{12}+p_{22}+\ldots+p_{52}=1 ; \\
& \vdots \\
& p_{15}+p_{25}+\ldots+p_{55}=1 ; \\
& p_{i l} \in\{0,1\}, i, l=1,2, \ldots, 5 .
\end{aligned}
$$

Solving above problem, $S^{*}=2$ is obtained. Substituting the elements of $\Pi^{\prime}(S)$, the problem in Eq. (13) is formulated and $V^{*}=1.033$ is attained. Eventually, the goal programming model of Eq. (14) is formulated as below:

$$
\begin{aligned}
& \min d_{1}^{-}+d_{2}^{-} \\
& \text {S.T. } \\
& 0.1 p_{12}+0.1 p_{13}+\ldots+0.4 p_{54}+0.2 p_{55}+d_{1}^{-}-d_{1}^{+}=2 \\
& 0.1 p_{11}+0.216 p_{12}+\ldots+0.2 p_{51}+0.2 p_{52}+d_{2}^{-}-d_{2}^{+}=1.033 \\
& p_{11}+p_{12}+\ldots+p_{15}=1 \\
& p_{21}+p_{22}+\ldots+p_{25}=1 \\
& p_{31}+p_{32}+\ldots+p_{35}=1 \\
& p_{41}+p_{42}+\ldots+p_{45}=1 \\
& p_{51}+p_{52}+\ldots+p_{55}=1 \\
& p_{i l} \in\{0,1\}, i, l=1,2, \ldots, 5
\end{aligned}
$$

Solving this model, the optimal solution is obtained as $p_{15}^{*}=p_{23}^{*}=p_{31}^{*}=p_{42}^{*}=p_{54}^{*}=1$. On the basis of this result, the ranking of alternatives is $m_{3}>m_{4}>m_{2}>m_{5}>m_{1}$. Liao et al. (2014) solved this problem employing different distance functions among HFLTSs. Applying 
generalized weighted distance measure, generalized weighted Hausdorff distance measure, and generalized hybrid weighted distance measure with $\lambda=1$, a similar ranking was obtained. Nonetheless, for different distance measures with $\lambda=2,4,6,10$, the ranking was $m_{3}>m_{2}>m_{4}>m_{5}>m_{1}$. It is notable that the results obtained with the proposed method completely coincide with Liao et al. (2014) different distance measures when $\lambda=1$, while there are small differences with other values of $\lambda$.

Solving the above problem by different methods, the following results are remarkable.

(1) Rodríguez et al. (2012) method includes three phases: (1) transformation, (2) aggregation, and (3) exploitation. Applying min-upper and max-lower operators, for five alternatives it follows that $H^{\prime}\left(m_{1}\right)=\left\{s_{0}, s_{1}\right\}, H^{\prime}\left(m_{2}\right)=\left\{s_{1}\right\}, H^{\prime}\left(m_{3}\right)=\left\{s_{2}\right\}$, $H^{\prime}\left(m_{4}\right)=\left\{s_{1}\right\}$, and $H^{\prime}\left(m_{5}\right)=\left\{s_{0}\right\}$. The preference degrees among these intervals show that $m_{3}>m_{2} \sim m_{4}>m_{1}>m_{5}$.

(2) The idea of likelihood-based comparison of Lee and Chen (2015), first the 1-cut of $\sigma$ is $h(\sigma(1))=\left[s_{-3}, s_{3}\right]$. Letting $h\left(m_{i j}(1)\right)$ be the 1 -cut of alternative $m_{i}$ in criterion $c_{j}$, then $h\left(m_{11}(1)\right)=\left[s_{-2}, s_{0}\right], \quad h\left(m_{12}(1)\right)=\left[s_{0}, s_{1}\right], \quad h\left(m_{13}(1)\right)=\left[s_{0}, s_{2}\right]$, $h\left(m_{14}(1)\right)=\left[s_{1}, s_{2}\right], h\left(m_{21}(1)\right)=\left[s_{0}, s_{2}\right], h\left(m_{22}(1)\right)=\left[s_{1}, s_{2}\right], h\left(m_{23}(1)\right)=\left[s_{0}, s_{1}\right]$, $h\left(m_{24}(1)\right)=\left[s_{0}, s_{2}\right], h\left(m_{31}(1)\right)=\left[s_{2}, s_{3}\right], h\left(m_{32}(1)\right)=\left[s_{1}, s_{3}\right], h\left(m_{33}(1)\right)=\left[s_{1}, s_{2}\right]$, $h\left(m_{34}(1)\right)=\left[s_{2}, s_{2}\right], h\left(m_{41}(1)\right)=\left[s_{0}, s_{2}\right], h\left(m_{42}(1)\right)=\left[s_{-1}, s_{1}\right], h\left(m_{43}(1)\right)=\left[s_{1}, s_{\#}\right]$, $h\left(m_{44}(1)\right)=\left[s_{1}, s_{2}\right], h\left(m_{51}(1)\right)=\left[s_{-1}, s_{0}\right], h\left(m_{52}(1)\right)=\left[s_{0}, s_{2}\right], h\left(m_{53}(1)\right)=\left[s_{0}, s_{2}\right]$, and $h\left(m_{54}(1)\right)=\left[s_{0}, s_{1}\right]$. Based on the HFLWA operator of Lee and Chen (2015), the score of alternatives are obtained as $R\left(m_{1}\right)=0.5525, R\left(m_{2}\right)=0.6352, R\left(m_{3}\right)=0.8107$, $R\left(m_{4}\right)=0.6531$, and $R\left(m_{5}\right)=0.5442$, respectively; thus, $m_{3}>m_{4}>m_{2}>m_{1}>m_{5}$.

(3) Using hesitant linguistic weighted average (HLWA) operator of Wei et al. (2014), $\operatorname{HLWA}\left(m_{1}\right)=\left\{s_{-1}, s_{0}, s_{1}\right\}, \quad \operatorname{HLWA}\left(m_{2}\right)=\left\{s_{0}, s_{1}, s_{2}\right\}, \quad \operatorname{HLWA}\left(m_{3}\right)=\left\{s_{1}, s_{2}\right\}$, $\operatorname{HLWA}\left(m_{4}\right)=\left\{s_{0}, s_{1}, s_{2}\right\}$, and $\operatorname{HLWA}\left(m_{5}\right)=\left\{s_{0}, s_{1}\right\}$. The alternatives are ranked as $m_{3}>m_{2} \sim m_{4}>m_{5}>m_{1}$.

Considering these methods, it is obvious that $m_{3}$ ranked first employing all methods. Nevertheless, Rodríguez et al. (2012) and Wei et al. (2014) methods determined $m_{2}$ and $m_{4}$ as indifferent. The only difference ranking between the proposed method and Lee and Chen (2015) are $m_{5}$ and $m_{1}$.

Farhadnia (Farhadnia 2015) moreover analyzed the above problem assuming that criteria weight vector is unknown. He determined criteria weight vector using different distances. Using generalized distance with $\lambda=0.5$. This vector is obtained as $W_{1}=(0.2322,0.2343,0.2444,0.2891)$. When $\lambda=1$, the weight vector is $W_{2}=(0.2483,0.2345,0.2483,0.2690)$ and for $\lambda=2$, this vector is $W_{3}=(0.2558,0.2352,0.2522,0.2569)$. Applying these weights, the proposed method ranks alternatives as $m_{3}>m_{4}>m_{2}>m_{5}>m_{1}$. Accordingly, the results seem stable regard to small changes in criteria weights. It can be considered as an advantage of the proposed method, beyond its other advantages as summarized in the conclusion. 


\section{A real world application}

To illustrate a real world application, one of the most important decisions in the production of topographic maps has been considered. Topographic maps show the position of natural and man-made features on the ground. These maps are reliable sources of spatial date and are widely used in construction and development projects.

To prepare topographic maps the ground data should be collected from the earth. The data gathering process for large scale maps of narrow fields can be done by land survey operation; even though, it's not economic way for producing topographic maps of a broad land in terms of time and cost. Under these circumstances, the producers employ aerial photography by a specific camera.

Choosing the right camera is a key decision, affects the quality and accuracy of the images, and the cost of the operation. Although the optimal performance of the aerial camera depends on some factors like the flight altitude, intended use of the maps, and also condition of the ground in terms of the complexity and density of features; nonetheless, available camera in the market can be compared and evaluated by specified and common criteria. In this research, nine cameras are evaluated with regards to eight criteria. The constructed decision matrix is shown in Table 6.

Table 6. Decision matrix of camera selection

\begin{tabular}{|l|c|c|c|c|c|c|c|c|}
\hline Brand name & $\begin{array}{c}\text { Number } \\
\text { of pixels }\end{array}$ & $\begin{array}{c}\text { Pixel } \\
\text { size } \\
(\mu \mathrm{m})\end{array}$ & $\begin{array}{c}\text { Focal } \\
\text { length } \\
(\mathrm{mm})\end{array}$ & $\begin{array}{c}\text { Frame } \\
\text { rate } \\
(\text { second })\end{array}$ & $\begin{array}{c}\text { Color } \\
\text { depth } \\
\text { (bit per } \\
\text { color })\end{array}$ & FMC & $\begin{array}{c}\text { After sale } \\
\text { service }\end{array}$ & $\begin{array}{c}\text { Ease of } \\
\text { use }\end{array}$ \\
\hline $\begin{array}{l}\text { Z/I Imaging } \\
\text { DMC II }\end{array}$ & $\begin{array}{c}16,768^{*} \\
14,016\end{array}$ & 5.6 & 112 & 2.3 & 12 & Yes & $\left\{s_{4}, s_{5}\right\}$ & $\left\{s_{5}, s_{6}\right\}$ \\
\hline $\begin{array}{l}\text { VexcelUltra- } \\
\text { Cam-D }\end{array}$ & $\begin{array}{c}11,500^{*} \\
7,500\end{array}$ & 9 & 100 & 1 & $>12$ & Yes & $\left\{s_{2}, s_{3}\right\}$ & $\left\{s_{4}, s_{5}\right\}$ \\
\hline $\begin{array}{l}\text { VexcelUltra- } \\
\text { Cam_X }\end{array}$ & $\begin{array}{c}14,430^{*} \\
9,420\end{array}$ & 7.2 & 100 & 1.35 & $>12$ & Yes & $\left\{s_{2}, s_{3}, s_{4}\right\}$ & $\left\{s_{3}\right\}$ \\
\hline $\begin{array}{l}\text { VexcelUltra- } \\
\text { Cam_XP }\end{array}$ & $\begin{array}{c}17,310^{*} \\
11,310\end{array}$ & 6 & 100 & 2 & $>12$ & Yes & $\left\{s_{3}, s_{4}\right\}$ & $\left\{s_{4}, s_{5}\right\}$ \\
\hline $\begin{array}{l}\text { VexcelUltra- } \\
\text { Cam_Eagle }\end{array}$ & $\begin{array}{c}20,010^{*} \\
13,080\end{array}$ & 5.2 & 80,210 & 1.8 & $>12$ & Yes & $\left\{s_{5}, s_{6}\right\}$ & $\left\{s_{4}\right\}$ \\
\hline DiMAC & $\begin{array}{c}10,500^{*} \\
7,200\end{array}$ & 6.8 & $\begin{array}{c}55,80, \\
100,120\end{array}$ & 1.9 & 16 & Yes & $\left\{s_{4}, s_{5}, s_{6}\right\}$ & $\left\{s_{2}, s_{3}, s_{4}\right\}$ \\
\hline $\begin{array}{l}\text { Applanix } \\
\text { DSS }\end{array}$ & $\begin{array}{c}5,436^{*} \\
4,092\end{array}$ & 9 & 55 or 35 & $<3$ & 12 & $\begin{array}{c}\text { No, not } \\
\text { required }\end{array}$ & $\left\{s_{2}, s_{3}, s_{4}\right\}$ & $\left\{s_{2}, s_{3}\right\}$ \\
\hline $\begin{array}{l}\text { IGI DIGI- } \\
\text { CAM }\end{array}$ & $\begin{array}{c}7,216^{*} \\
5,412\end{array}$ & 6.8 & $\begin{array}{c}28,35,80, \\
100,150, \\
210,300\end{array}$ & 1.9 & 14 & Yes & $\left\{s_{6}\right\}$ & $\left\{s_{5}, s_{6}\right\}$ \\
\hline $\begin{array}{l}\text { RolleiMetric } \\
\text { AIC }\end{array}$ & $\begin{array}{c}\text { Up to } \\
7,228^{*} \\
5,428\end{array}$ & 6.8 & $\begin{array}{c}35,47, \\
60,72\end{array}$ & N/A & 16 & N/A & $\left\{s_{3}, s_{4}\right\}$ & $\left\{s_{6}\right\}$ \\
\hline
\end{tabular}


In the above table,

- "Number of pixels" stands for the dimension of each digital image frame.

- "Pixel size" is the physical dimension of any individual pixel in a CCD.

- "Frame rate" illustrates the time delay between any two consecutive images captured in an aerial photography mission.

- "Color depth" is the number of bits used to indicate the color of a single pixel.

- FMC is an acronym for Forward Motion Compensation. It's a technique employed to compensate image blurring occurring as a result of slower shutter speeds, faster flight speeds and so on.

Considering HFLTS matrix in Table 6, the score and variance decision matrix are obtained as illustrated in Tables 7 and 8, respectively.

Table 7. Score function decision matrix of camera selection

\begin{tabular}{|l|c|c|c|c|c|c|c|c|}
\hline Brand name & $\begin{array}{c}\text { Number } \\
\text { of pixels }\end{array}$ & $\begin{array}{c}\text { Pixel size } \\
(\mu \mathrm{m})\end{array}$ & $\begin{array}{c}\text { Focal } \\
\text { length } \\
(\mathrm{mm})\end{array}$ & $\begin{array}{c}\text { Frame } \\
\text { rate } \\
(\text { second) }\end{array}$ & $\begin{array}{c}\text { Color } \\
\text { depth } \\
\text { (bit per } \\
\text { color })\end{array}$ & FMC & $\begin{array}{c}\text { After } \\
\text { sale ser- } \\
\text { vice }\end{array}$ & $\begin{array}{c}\text { Ease of } \\
\text { use }\end{array}$ \\
\hline $\begin{array}{l}\text { Z/I Imaging } \\
\text { DMC II } 250\end{array}$ & $\begin{array}{c}16,768^{*} \\
14,016\end{array}$ & 5.6 & 112 & 2.3 & 12 & 1 & 4.5 & 5.5 \\
\hline $\begin{array}{l}\text { VexcelUltra- } \\
\text { Cam-D }\end{array}$ & $\begin{array}{c}11,500^{*} \\
7,500\end{array}$ & 9 & 100 & 1 & $>12$ & 1 & 2.5 & 4.5 \\
\hline $\begin{array}{l}\text { VexcelUltra- } \\
\text { Cam_X }\end{array}$ & $\begin{array}{c}14,430^{*} \\
9,420\end{array}$ & 7.2 & 100 & 1.35 & $>12$ & 1 & 3 & 3 \\
\hline $\begin{array}{l}\text { VexcelUltra- } \\
\text { Cam_XP }\end{array}$ & $\begin{array}{c}17,310^{*} \\
11,310\end{array}$ & 6 & 100 & 2 & $>12$ & 1 & 3.5 & 4.5 \\
\hline $\begin{array}{l}\text { VexcelUltra- } \\
\text { Cam_Eagle }\end{array}$ & $\begin{array}{c}20,010^{*} \\
13,080\end{array}$ & 5.2 & 145 & 1.8 & $>12$ & 1 & 5.5 & 4 \\
\hline DiMAC & $\begin{array}{c}10,500^{*} \\
7,200\end{array}$ & 6.8 & 88.75 & 1.9 & 16 & 1 & 5 & 3 \\
\hline $\begin{array}{l}\text { Applanix } \\
\text { DSS }\end{array}$ & $\begin{array}{c}5,436^{*} \\
4,092\end{array}$ & 9 & 45.00 & $<3$ & 12 & 0 & 3 & 2.5 \\
\hline $\begin{array}{l}\text { IGI DIGI- } \\
\text { CAM }\end{array}$ & $\begin{array}{c}7,216^{*} \\
5,412\end{array}$ & 6.8 & 119.13 & 1.9 & 14 & 1 & 6 & 5.5 \\
\hline $\begin{array}{l}\text { RolleiMetric } \\
\text { AIC }\end{array}$ & $\begin{array}{c}\text { Up to } \\
7,228^{*} \\
5,428\end{array}$ & 6.8 & 53.50 & N/A & 16 & 0 & 3.5 & 6 \\
\hline
\end{tabular}

Table 8. Variance function matrix of camera selection

\begin{tabular}{|l|c|c|c|c|c|c|c|c|}
\hline \multicolumn{1}{|c|}{ Brand name } & $\begin{array}{c}\text { Number } \\
\text { of pixels }\end{array}$ & $\begin{array}{c}\text { Pixel } \\
\text { size } \\
(\mu \mathrm{m})\end{array}$ & $\begin{array}{c}\text { Focal } \\
\text { length } \\
(\mathrm{mm})\end{array}$ & $\begin{array}{c}\text { Frame } \\
\text { rate }(\mathrm{se}- \\
\text { cond) }\end{array}$ & $\begin{array}{c}\text { Color depth } \\
(\text { bit per } \\
\text { color })\end{array}$ & FMC & $\begin{array}{c}\text { After } \\
\text { sale } \\
\text { service }\end{array}$ & $\begin{array}{c}\text { Ease of } \\
\text { use }\end{array}$ \\
\hline $\begin{array}{l}\text { Z/I Imaging DMC } \\
\text { II }_{250}\end{array}$ & 0.0 & 0.0 & 0 & 0.0 & 0.0 & 0.0 & 0.5 & 0.5 \\
\hline VexcelUltraCam-D & 0.0 & 0.0 & 0 & 0.0 & 0.0 & 0.0 & 0.5 & 0.5 \\
\hline VexcelUltraCam_X & 0.0 & 0.0 & 0 & 0.0 & 0.0 & 0.0 & 0.82 & 0 \\
\hline
\end{tabular}


End of Table 8

\begin{tabular}{|l|c|c|c|c|c|c|c|c|}
\hline \multicolumn{1}{|c|}{ Brand name } & $\begin{array}{c}\text { Number } \\
\text { of pixels }\end{array}$ & $\begin{array}{c}\text { Pixel } \\
\text { size } \\
(\mu \mathrm{m})\end{array}$ & $\begin{array}{c}\text { Focal } \\
\text { length } \\
(\mathrm{mm})\end{array}$ & $\begin{array}{c}\text { Frame } \\
\text { rate (se- } \\
\text { cond) }\end{array}$ & $\begin{array}{c}\text { Color depth } \\
(\text { bit per } \\
\text { color })\end{array}$ & FMC & $\begin{array}{c}\text { After } \\
\text { sale } \\
\text { service }\end{array}$ & $\begin{array}{c}\text { Ease of } \\
\text { use }\end{array}$ \\
\hline VexcelUltraCam_XP & 0.0 & 0.0 & 0 & 0.0 & 0.0 & 0.0 & 0.5 & 0.5 \\
\hline $\begin{array}{l}\text { VexcelUltraCam_ } \\
\text { Eagle }\end{array}$ & 0.0 & 0.0 & 91.92 & 0.0 & 0.0 & 0.0 & 0.5 & 0 \\
\hline DiMAC & 0.0 & 0.0 & 27.80 & 0.0 & 0.0 & 0.0 & 0.82 & 0.82 \\
\hline Applanix DSS & 0.0 & 0.0 & 14.14 & 0.0 & 0.0 & 0.0 & 0.82 & 0.5 \\
\hline IGI DIGICAM & 0.0 & 0.0 & 95.61 & 0.0 & 0.0 & 0.0 & 0 & 0.5 \\
\hline RolleiMetric AIC & 0.0 & 0.0 & 256.33 & 0.0 & 0.0 & 0.0 & 0.5 & 0 \\
\hline
\end{tabular}

Considering Tables 7 and 8, the following two matrices are constructed.

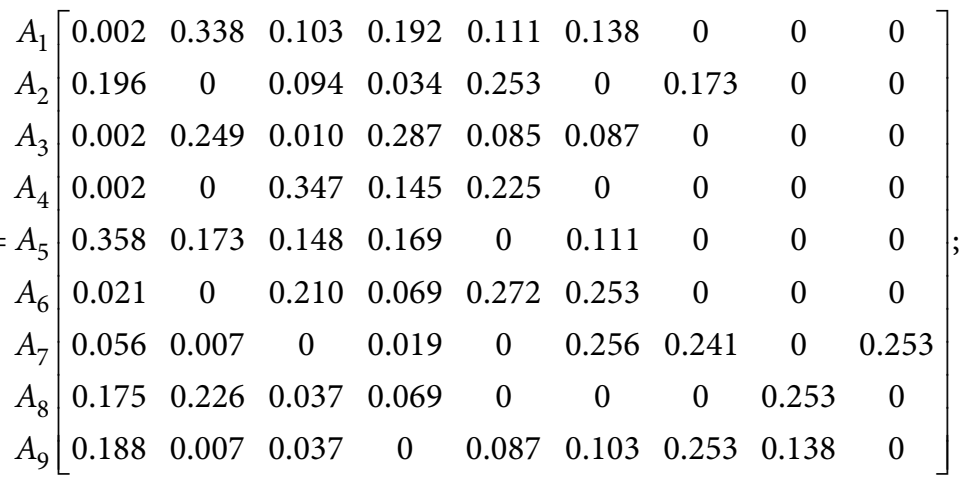

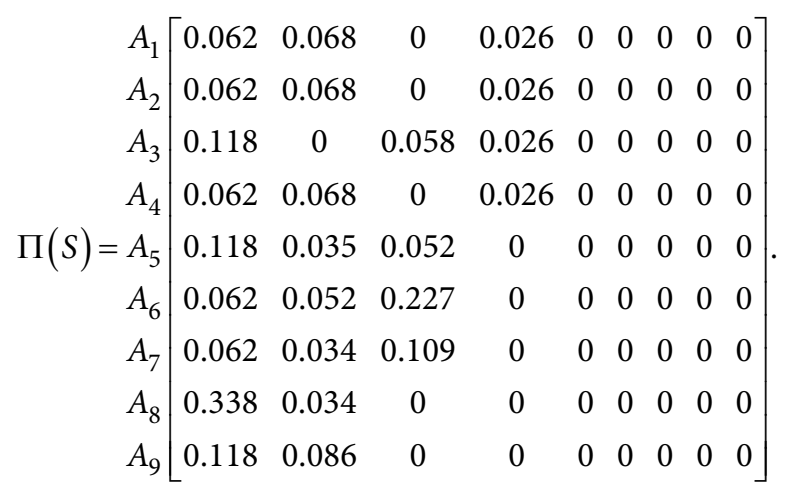

Formulating and solving the problems in Eqs (12) and (13), the results are found to be $S^{*}=2.595$ and $V^{*}=0.677$. The goal programming model of Eq. (14) is then formulated as: 


$$
\begin{aligned}
& \min d_{1}^{-}+d_{2}^{-} \\
& S . T . \\
& 0.002 p_{11}+0.338 p_{12}+\ldots+0.138 p_{98}+0 p_{99}+d_{2}^{-}-d_{2}^{+}=2.595 \\
& 0.062 p_{11}+0.068 p_{12}+\ldots+0 p_{98}+0 p_{99}+d_{2}^{-}-d_{2}^{+}=0.677 \\
& p_{11}+p_{12}+\ldots+p_{19}=1 \\
& p_{21}+p_{22}+\ldots+p_{29}=1 \\
& \vdots \\
& p_{91}+p_{92}+\ldots+p_{99}=1 \\
& p_{11}+p_{21}+\ldots+p_{91}=1 \\
& p_{12}+p_{22}+\ldots+p_{92}=1 \\
& \vdots \\
& p_{19}+p_{29}+\ldots+p_{99}=1 \\
& p_{i l} \in\{0,1\}, i, l=1,2, \ldots, 9
\end{aligned}
$$

Solving this model, the optimal solution obtained as $p_{12}^{*}=p_{25}^{\star}=p_{34}^{\star}=p_{43}^{*}=$ $p_{51}^{*}=p_{66}^{*}=p_{79}^{*}=p_{88}^{*}=p_{97}^{*}=1$. Based on these results, the ranking of alternatives eventuates as $A_{5}>A_{1}>A_{4}>A_{3}>A_{2}>A_{6}>A_{9}>A_{8}>A_{7}$. Consequently, according to the proposed method, the best camera is "Vexcel Ultra Cam_Eagle"; furthermore, "Z/I Imaging $\mathrm{DMC} \mathrm{II}_{250}$ " and "Vexcel Ultra Cam_XP" gain the $2^{\text {nd }}$ and $3^{\text {rd }}$ ranks.

\section{Qualitative analysis}

In the previous section, a problem was solved considering the scheduled method and the obtained results are compared with other extended procedures. In view of the features of a good decision making method proposed by Saaty and Ergu (2015), the following conclusions can be made regarding our recommended method.

(1) Simplicity of execution. Considering ease of use as a criterion for choosing an MADM method, the proposed method seems an appropriate tool without any unrecognizable or difficult step. However, the amount of computations required is somewhat high. Therefore, the method rates medium in this feature.

(2) Comprehensive structure: breadth and depth. Saaty and Ergu (2015) called an MADM method as broad if it contains a number of distinct criteria and deep if criteria can broke down to sub-criteria. It is clear that the proposed method is broad since it contains several distinct criteria. However, its depth feature can be criticized.

(3) Comprehensive structure consisting of merit substructures. A decision structure is comprehensive if it represents a decision problem considering different political, social, economic, legal, and etc. criteria. Considering the proposed method, there isn't any limitation to define different criteria. 
(4) Logical, mathematical procedure. The proposed method follows a logical procedure acquiring from linear assignment method.

(5) Justification of the approach - justifiable axioms. The main features of the proposed method are similar to linear assignment method as a well-known and widely accepted method.

(6) Scales of measurement. The information of the problem is assumed to be in the form of HFLTS.

(7) Synthesis of judgments with merging functions. For group decision making, the individual decision matrices can be synthesized. Hence, according to Saaty and Ergu (2015), the proposed method gains medium in this criterion.

(8) Ranking of tangibles. Since the arranged method provides a cardinal ranking of alternatives, it is ranked high in this criterion.

(9) Generalization to ranking of intangibles. The advised method rated high in this criterion since the intangible factors are evaluated using HFLTSs with a great flexibility in translating linguistic variables.

(10) Rank preservation and reversal. Considering non-existence of an exact solution for uncertain problems in one hand and possibility of sensitivity analysis in the designed method, this feature is evaluated as medium for the proposed method.

(11) Sensitivity analysis. The determined method includes criteria weighting vector and alternatives performance as its input parameters. Sensitivity analysis is applicable to assess the impact of attribute weights change on ranking of alternatives. Thus, it rates medium in this criterion.

(12) Validation of decision problems. The expected method is performable in realworld problems with tangible and intangible factors.

(13) Generalizability to dependence and feedback. The method doesn't consider any treat regarding criteria dependence.

(14) Applicability to conflict resolution. As described earlier, decision makers can persuade each other to resolve the conflict and if they don't achieve an agreement, their assessments are combined in the form of a HFLTS. Therefore, the proposed method rates high in this feature.

(15) Prediction of the outcome of decisions with intangibles. As previously mentioned, the proposed method uses advanced and formalized framework of HFLTSs to deal with intangibles. The power of HFLTS provides a method of predicting preferences of decision makers. Ergo, the method rates medium in this feature.

(16) Trustworthiness and validity of the approach. The planned method works with cardinal measurements with a mathematical logical procedure. Resultantly, it rates high in this criterion.

Performance of the proposed method on above mentioned criteria are summarized in Table 9. 
Table 9. Summarizes the performance of the proposed method regard to the above criteria

\begin{tabular}{|c|l|c|}
\hline & \multicolumn{1}{|c|}{ Criterion } & $\begin{array}{c}\text { The proposed method } \\
\text { is rated }\end{array}$ \\
\hline 1 & Simplicity of execution & Medium \\
\hline 2 & Comprehensive structure: breadth and depth & Medium \\
\hline 3 & Comprehensive structure consisting of merit substructures & High \\
\hline 4 & Logical, mathematical procedure & High \\
\hline 5 & Justification of the approach - justifiable axioms & High \\
\hline 6 & Scales of measurement & Medium \\
\hline 7 & Synthesis of judgments with merging functions & High \\
\hline 8 & Ranking of tangibles & High \\
\hline 9 & Generalization to ranking of intangibles & Medium \\
\hline 10 & Rank preservation and reversal & High \\
\hline 11 & Sensitivity analysis & Low \\
\hline 12 & Validation of decision problems & High \\
\hline 13 & Generalizability to dependence and feedback & Medium \\
\hline 14 & Applicability to conflict resolution & High \\
\hline 15 & Prediction of the outcome of decisions with intangibles & \\
\hline 16 & Trustworthiness and validity of the approach &
\end{tabular}

Taking into account the above table and its applications, it seems that the considered method is an acceptable method of decision making under uncertainty, both practically and theoretically.

\section{Conclusions}

Linguistic variables improve the ability of decision makers in expressing their opinions with linguistic terms instead of quantitative values. Ordinal fuzzy sets allow experts to express their judgments using a single term, while it is possible that experts hesitate among several values. In fact, terms such as "below medium" or "medium to good" are common in human recognition. This hesitancy is allowed in hesitant fuzzy linguistic term sets; therefore, these sets have played a significant role in human decision making. Several methods are previously proposed dealing multi-criteria decision making with HFLTSs. In this paper, linear assignment method is extended for multi-criteria group decision making when experts opinions are expressed by HFLTSs. After aggregation of individual decision matrices in a group decision matrix, using the concept of HFLTS score and variance functions, the HFLTSs based MCDM problem is converted to a bi-objective linear assignment method for finding the best ranking of alternatives. This multi objective problem is then solved with goal programming. Comparison of the results of the contemplated method proved its applicability in MCDM problems while it produced results consistent with previous methods.

The algorithmic scheme of the proposed method can facilitate its application in real world decision problems. Furthermore, the mathematical problem of the method can be easily 
solved with the current package, e.g. Lingo. The prominent advantages of the proposed method consist of (1) great computational efficiency and simplicity, (2) logical and well-known mathematical background, (3) requiring least parameters to be chosen by experts that can influence the obtained results, (4) applicable to solve decision making problems with mix data including exact and ambiguous information (as represented in the real world application of Sec. 5) while other methods required all data to be in the form of HFLTS. All in all, compared with computational difficulty of previously presented methods, the proposed linear assignment based method can be known as suitable for decision making with hesitant information.

\section{References}

Abdolazimi, A.; Momeni, M.; Montazeri, M. 2015. Comparing ELECTRE and linear assignment methods in zoning shahroud-bastam watershed for artificial recharge of groundwater with GIS technique, Modern Applied Science 9(1): 68-82. https://doi.org/10.5539/mas.v9n1p68

Atanassov, K. 1986. Intuitionistic fuzzy sets, Fuzzy Sets and Systems 20(1): 87-96. http://dx.doi.org/10.1016/S0165-0114(86)80034-3

Atanassov, K.; Gargov, G. 1989. Interval valued intuitionistic fuzzy sets, Fuzzy Sets and Systems 31(3): 343-349. https://doi.org/10.1016/0165-0114(89)90205-4

Bashiri, M.; Badri, H.; Hejazi, T. H. 2011. Selecting optimum maintenance strategy by fuzzy interactive linear assignment method, Applied Mathematical Modeling 35(1): 152-164. https://doi.org/10.1016/j.apm.2010.05.014

Baykasoğlu, A.; Subulan, K.; Karaslan, F. S. 2016. A new fuzzy linear assignment method for multiattribute decision making with an application to spare parts inventory classification, Applied Soft Computing 42: 1-17. https://doi.org/10.1016/j.asoc.2016.01.031

Bellman, R. E.; Zadeh, L. A. 1970. Decision-making in a fuzzy environment, Management Science 17(4): 141-164. https://doi.org/10.1287/mnsc.17.4.B141

Bernardo, J. J.; Blin, J. M. 1977. A programming model of consumer choice among multi-attributed brands, Journal of Consumer Research 4(2): 111-118. https://doi.org/10.1086/208686

Chen, T. Y. 2013. A linear assignment method for multiple-criteria decision analysis with interval type2 fuzzy sets, Applied Soft Computing 13(5): 2735-2748. https://doi.org/10.1016/j.asoc.2012.11.013

Chen, T. Y. 2014. The extended linear assignment method for multiple criteria decision analysis based on interval-valued intuitionistic fuzzy sets, Applied Mathematical Modeling 38(7-8): 2101-2117. https://doi.org/10.1016/j.apm.2013.10.017

Farhadnia, B. 2015. Multiple criteria decision-making methods with completely unknown weights in hesitant fuzzy linguistic term setting, Knowledge-Based Systems 93: 135-144.

https://doi.org/10.1016/j.knosys.2015.11.008

Ginevičius, R. 2011. A new determining method for the criteria weights in multi-criteria evaluation, International Journal of Information Technology \& Decision Making 10(6): 1067-1095. https://doi.org/10.1142/S0219622011004713

Grattan-Guinness, I. 1976. Fuzzy membership mapped onto interval and many-valued quantities, Mathematical Logic Quarterly 22(1): 149-160. https://doi.org/10.1002/malq.19760220120

Herrera, F.; Herrera-Viedma, E.; Verdegay, J. L. 1996. A model of consensus in group decision making under linguistic assessments, Fuzzy Sets Systems 78(1): 73-87. https://doi.org/10.1016/0165-0114(95)00107-7

Herrera, F.; Martinez, L. 2000. A 2-tuple fuzzy linguistic representation model for computing with words, IEEE Transactions on Fuzzy Systems 8(6): 746-752. https://doi.org/10.1109/91.890332 
Hwang, C. L.; Yoon. K. P. 1995. Multiple attribute decision making: an introduction. Sage Publication Inc.: California. https://doi.org/10.1016/j.ins.2014.09.061

Lee, L. W.; Chen, S. M. 2015. Fuzzy decision making based on likelihood-based comparison relations of hesitant fuzzy linguistic term sets and hesitant fuzzy linguistic operators, Information Sciences 294: 513-529. https://doi.org/10.1016/j.ins.2014.09.061

Liang, Q.; Mendel, J. M. 2000. Interval type-2 fuzzy logic systems: theory and design, IEEE Transactions on Fuzzy Systems 8(5): 535-550. https://doi.org/10.1109/91.873577

Liao, H.; Xu, Z. 2015. Approaches to manage hesitant fuzzy linguistic information based on the cosine distance and similarity measures for HFLTSs and their application in qualitative decision making, Expert Systems with Applications 42: 5328-5336. https://doi.org/10.1016/j.eswa.2015.02.017

Liao, H.; Xu, Z.; Zeng, X. J. 2014. Distance and similarity measures for hesitant fuzzy linguistic term sets and their application in multi-criteria decision making, Information Sciences 271: 125-142. https://doi.org/10.1016/j.ins.2014.02.125

Liao, H.; Xu, Z.; Zeng, X. J. 2015a. Hesitant fuzzy linguistic VIKOR method and its application in qualitative multiple criteria decision making, IEEE Transactions on Fuzzy Systems 23: 1343-1355. https://doi.org/10.1109/TFUZZ.2014.2360556

Liao, H.; Xu, Z.; Zeng, X. J.; Merigó, J. M. 2015b. Qualitative decision making with correlation coefficients of hesitant fuzzy linguistic term sets, Knowledge-Based Systems 76: 127-138. https://doi.org/10.1016/j.knosys.2014.12.009

Lin, C. J.; Wen, U. P. 2004. A labeling algorithm for the fuzzy assignment problem, Fuzzy Sets and Systems 142(3): 20053-391. http://dx.doi.org/10.1016/S0165-0114(03)00017-4

Liu, H.; Rodríguez, R. M. 2014. A fuzzy envelope for hesitant fuzzy linguistic term set and its application to multi-criteria decision making, Information Sciences 258: 220-228. https://doi.org/10.1016/j.ins.2013.07.027

Liu, S. F.; Lin, Y. 2011. Grey systems theory and application. Heidelberg: Springer. https://doi.org/10.1007/978-3-642-16158-2

Martinez, L.; Herrera, F. 2010. An overview on the 2-tuple linguistic model for computing with words in decision making: extensions, applications and challenges, Information Science 207: 1-18. https://doi.org/10.1016/j.ins.2012.04.025

Montes, R.; Sánchez, A. M.; Villar, P.; Herrera, F. 2015. A web tool to support decision making in the housing market using hesitant fuzzy linguistic term sets, Applied Soft Computing 35: 949-957. https://doi.org/10.1016/j.asoc.2015.01.030

Razavi Hajiagha, S. H.; Amoozad Mahdiraji, H.; Hashemi, S. S. 2013. Multi-objective linear programming with interval coefficients: a fuzzy set based approach, Kybernetes 42(3): 482-496.

https://doi.org/10.1108/03684921311323707

Razavi Hajiagha, S. H.; Amoozad Mahdiraji, H.; Hashemi, S. S. 2014a. A hybrid model of fuzzy goal programming and grey numbers in continuous project time, cost, and quality tradeoff, International Journal of Advanced Manufacturing Technology 71(1-4): 117-126. https://doi.org/10.1007/s00170-013-5463-2

Razavi Hajiagha, S. H.; Amoozad Mahdiraji, H.; Hashemi, S. S. 2015. Determining weights of fuzzy attributes for multi-attribute decision-making problems based on consensus of expert opinions, Technological and Economic Development of Economy 21(5): 738-755.

Razavi Hajiagha, S. H.; Amoozad Mahdiraji, H.; Hashemi, S. S.; Zavadskas, E. K. 2014b. Fuzzy multi objective programming based on comprise VIKOR method, International Journal of Information Technology and Decision Making 13(4): 679-699. https://doi.org/10.3846/20294913.2015.1058301

Razavi Hajiagha, S. H.; Amoozad Mahdiraji, H.; Zavadskas, E. K.; Hashemi, S. S. 2014c. Maximizing and minimizaing sets in solving fuzzy linear programming, Economic Computation and Economic Cybernetics Studies and Research 48(2): 113-133. 
Rodríguez, R. M.; Martínez, L.; Herrera, F. 2012. Hesitant fuzzy linguistic terms sets for decision making, IEEE Transactions on Fuzzy Systems 20(1): 109-119. https://doi.org/10.1109/TFUZZ.2011.2170076

Rodríguez, R. M.; Martínez, L.; Herrera, F. 2013. A group decision making model dealing with comparative linguistic expressions based on hesitant fuzzy linguistic term set, Information Sciences 241: 28-42. https://doi.org/10.1016/j.ins.2013.04.006

Saaty, T. L. 1977. A scaling method for priorities in hierarchical structures, Journal of Mathematical Psychology 15(3): 234-281. https://doi.org/10.1016/0022-2496(77)90033-5

Saaty, T.; Ergu, D. 2015. When is a decision-making method trustworthy? criteria for evaluating multicriteria decision-making methods, International Journal of Information Technology and Decision Making 14(6): 1171-1188. https://doi.org/10.1142/S021962201550025X

Srinivasan, V.; Shocker, A. D. 1973. Linear programming techniques for multidimensional analysis of preferences, Psychometrika 38(3): 337-369. https://doi.org/10.1007/BF02291658

Torra, V. 2010. Hesitant fuzzy sets, International Journal of Intelligent Systems 25(6): 529-539. https://doi.org/10.1002/int.20418

Tzeng, G. H.; Huang, J. J. 2011. Multiple attribute decision making: methods and applications. Florida: CRC Press. https://doi.org/10.1201/b11032

Wang, H.; Xu, Z. 2015. Some consistency measures of extended hesitant fuzzy linguistic preference relations, Information Sciences 297: 316-331. https://doi.org/10.1016/j.ins.2014.10.047

Wang, J.; Wang, J. Q.; Zhang, H. Y. Chen, X. H. 2015. Multi-criteria decision-making based on hesitant fuzzy linguistic term sets: an outranking approach, Knowledge-Based Systems 86: 224-236. https://doi.org/10.1016/j.knosys.2015.06.007

Wei, C.; Ren, Z.; Rodríguez, R. M. 2015. A hesitant fuzzy linguistic TODIM method based on a score function, International Journal of Computational Intelligence Systems 8(4): 701-712. https://doi.org /10.1080/18756891.2015.1046329

Wei, C.; Zhao, N.; Tang, X. 2014. Operators and comparisons of hesitant fuzzy linguistic term sets, IEEE Transactions on Fuzzy Systems 22(3): 575-585. https://doi.org/10.1109/TFUZZ.2013.2269144

$\mathrm{Wu}, \mathrm{Z}$.; Xu, J. 2015. Managing consistency and consensus in group decision making with hesitant fuzzy linguistic preference relations, Omega 65: 28-4. https://doi.org/10.1016/j.omega.2015.12.005

Xia, M. M.; Xu, Z. S. 2011. Hesitant fuzzy information aggregation in decision making, International Journal of Approximate Reasoning 52(3): 395-407. https://doi.org/10.1016/j.ijar.2010.09.002

$\mathrm{Xu}, \mathrm{Z}$. S. 2004. Uncertain Multiple attribute decision making: methods and applications. Beijing: Tsinghua University Press. https://doi.org/10.1007/978-3-662-45640-8

$\mathrm{Xu}, \mathrm{Z}$. S. 2005. Deviation measures of linguistic preference relations in group decision making, Omega 33(3): 249-254. https://doi.org/10.1016/j.omega.2004.04.008

$\mathrm{Xu}, \mathrm{Z}$. S. 2012. Linguistic decision making: theory and methods. Beijing: Science Press. https://doi.org/10.1007/978-3-642-29440-2

Yu, P. L. 1990. Forming winning strategies: an integrated theory of habitual domains. Berlin: Springer. https://doi.org/10.1007/978-3-642-61295-4

Zadeh, L. A. 1965. Fuzzy sets, Information and Control 8(3): 338-353. https://doi.org/10.1016/S0019-9958(65)90241-X

Zadeh, L. A. 1975. The concept of a linguistic variable and its application to approximate reasoning - I, Information Sciences 8(3): 199-249. https://doi.org/10.1016/0020-0255(75)90036-5

Zamri, N.; Abdullah, L. 2015. A linear assignment method of simple additive weighting system in linear programming approach under interval type-2 fuzzy set concepts for MCDM problem, in H. Sulaiman, M. Othman, M. Othman, Y. Rahim, N. Pee (Eds.). Advanced computer and communication engineering technology. Lecture Notes in Electrical Engineering, vol 315. Springer, Cham, 833-842. https://doi.org/10.1007/978-3-319-07674-4_78 
Zhang, Z.; Wu, C. 2014. On the use of multiplicative consistency in hesitant fuzzy linguistic preference relations, Knowledge-Based Systems 72: 13-27. https://doi.org/10.1016/j.knosys.2014.08.026

Zhu, B.; Xu, Z. 2014. Consistency measures for hesitant fuzzy linguistic preference relations, IEEE Transactions on Fuzzy Systems 22: 35-45. https://doi.org/10.1109/TFUZZ.2013.2245136

Zhu, B.; Xu, Z. 2016. Extended hesitant fuzzy sets, Technological and Economic Development of Economy 22(1): 100-121. https://doi.org/10.3846/20294913.2014.981882

Zimmerman, H. J. 1987. Fuzzy sets, decision making, and expert systems. Boston: Kluwer Academic Publisher. https://doi.org/10.1007/978-94-009-3249-4 